\title{
SARS-CoV-2 Infektion bei Kindern und Jugendlichen
}

\section{Ein Literaturüberblick der AG Infektiologie der ÖGKJ'}

\begin{abstract}
_ Im Dezember 2019 kam es in der chinesischen Region Hubei zum gehäuften Auftreten von Pneumoniefällen unbekannter Ätiologie [1]. Als Ursache konnte rasch ein Betacoronavirus, welches im weiteren Verlauf die Bezeichnung SARS-CoV-2 erhielt, identifiziert werden. Aufgrund der zunehmenden weltweiten Verbreitung rief die WHO schließlich am 11.03.2020 die laufende Pandemie aus [2]. Mittlerweile gibt es weltweit mehr als 3,5 Millionen positiv getestete Personen und mehr als 250.000 bestätigte Todesfälle [3]. Umfassende Informationen zu COVID-19 bei Kindern werden erst langsam verfügbar.
\end{abstract}

\section{Häufigkeit und Schwere der Erkrankung bei Kindern}

Kinder erkranken wesentlich seltener als Erwachsene. Eine Auswertung der ersten knapp 45.000 labor-bestätigten COVID-Fälle in China zeigt, dass Kinder unter 10 Jahren nur 0,9\% (416 Kinder) und Kinder zwischen 10 und 19 Jahren nur 1,2 \% (549 Kinder) der Fälle ausgemacht haben [4]. Diese Auswertung bezieht sich nur auf Fälle, welche in der Regel aufgrund von (relevanter) Symptomatik getestet wurden. Wie viele Patienten (inkl. Kindern) erkrankt sind, aber aufgrund einer milden Symptomatik nicht getestet wurden, ist naturgemäß unbekannt. Todesfälle im Kindesalter sind grundsätzlich selten, beispielsweise sind bis Ende April in den schwer betroffenen Ländern Spanien und Italien vier Kinder unter 9 Jahren und 5 Kinder zwischen 10-19 Jahren verstorben [5, 6]. Bisherige Daten zu intensivpflichtigen, pädiatrischen $\mathrm{Pa}$ -

\footnotetext{
1 Stand 04.05.2020
}

tienten sind kaum vorhanden, daher sind Daten zu Grund- bzw. Begleiterkrankungen, Alter, Schwere oder Organmanifestationen bisher nicht ausreichend beschrieben $[7,8]$.

In einer größeren pädiatrischen Arbeit mit über 2000 Kindern, welche auch Verdachtsfälle inkludiert, waren Kinder aller Altersstufen betroffen. Ein signifikanter Geschlechterunterschied zeigte sich nicht. Zirka $4 \%$ der inkludierten Fälle verliefen asymptomatisch, und fast $90 \%$ zeigten einen milden oder moderaten Verlauf. Schwere Verläufe waren insgesamt selten $(6 \%)$, jedoch waren Kinder unter 1 Jahr am häufigsten von einem schweren Verlauf betroffen. Allerdings waren in dieser Altersgruppe knapp $80 \%$ der Fälle lediglich Verdachtsfälle (ohne SARS-CoV-2-Laborbestätigung), sodass die Autoren davon ausgehen, dass ein nicht unbeträchtlicher Teil dieser schweren Verläufe durch andere Viren (v.a. RSV) und nicht durch SARS-CoV-2 verursacht gewesen sein könnte [9].

\section{Vertikale Transmission}

Bisher gibt es keine ausreichenden Hinweise, dass SARS-CoV-2 vertikal übertragen wird. In zwei kleinen Fallkohorten mit je neun an COVID-19 erkrankten Mütter, wurden die Neugeborenen allesamt negativ getestet $[10,11]$. Ebenso war die Testung von Amnionflüssigkeit, Nabelschnurblut und Muttermilch negativ [10]. Jedoch zeigten sich in einer diesen Kohorten vermehrte fetale Komplikationen wie Frühgeburtlichkeit oder respiratorischer Stress, wobei der direkte Zusammenhang mit SARS-CoV-2 nicht geklärt ist. Ebenso könnten diese
Komplikationen eher indirekt durch die Erkrankung der Mutter (z. B. wegen frühzeitiger Sectio) als durch eine Infektion des Kindes erklärt werden [11]. So muss natürlich auf neonatalen Intensivstationen damit gerechnet werden, dass aufgrund einer SARSCoV-2-Erkrankung der Mutter eine prämature Entbindung indiziert wird und die Frühgeborenen behandelt werden müssen. In einem Fallbericht aus China wurde ein Neugeborenes einer SARS-CoV-2 positiven Mutter 36 Stunden nach der Geburt positiv auf SARS-CoV-2 getestet. Die Testung von Nabelschnurblut, Plazentagewebe und Muttermilch waren negativ. Es ist daher unklar, ob es sich um eine vertikale oder postpartale Übertragung handelt. Der klinische Verlauf des Säuglings war mild [12]. In einer Arbeit erfolgte bei sechs Müttern und Neugeborenen die Testung auf $\lg M$ und IgG Antikörper, wobei zwei Säuglinge erhöhte lgM Werte zeigten, welche in der Regel nicht diaplazentar auf das Kind übertragen werden. [13].

In der Muttermilch SARS-CoV-2 positiver Mütter wurde bisher keine Virus-RNA nachgewiesen [10]. Von den meisten Fachgesellschaften wird das Stillen durch SARS-CoV-2 positive Mütter (bzw. Füttern abgepumpter Mutter-Milch) empfohlen. Dabei sollte aber in jedem Fall das Übertragungsrisiko durch Tröpfcheninfektion berücksichtigt werden (Mundschutz und Händehygiene).

\section{Klinik}

Der Großteil der Erkrankungen verläuft mild. Asymptomatische Verläufe sind beschrieben, wobei die Häufigkeit dieser noch unklar ist. Nach chine- 
sischen Daten entwickelten $75 \%$ der initial asymptomatischen Patienten im Verlauf Symptome, sodass durchwegs asymptomatische Verläufe derzeit als eher selten angesehen werden (geschätzte 1-3\%) [14]. Bei Kindern sind asymptomatische Verläufe in bis zu $16 \%$ der Gesamtkohorte [8] und in einzelnen Altersgruppen sogar in über $30 \%$ beschrieben [9], wobei hier teilweise unklar ist, ob es im weiteren Verlauf zu Symptomen gekommen ist.

Bei den wenigen detaillierten Berichten über spezifische Symptome bei Kindern mit COVID-19 wird Fieber in $40-100 \%$ und Husten in 40-100\% der symptomatischen Fälle beschrieben [7, 8, 15-19]. Weitere beschriebene Symptome sind Pharyngitis (5-40\%), Rhinitis (10-30\%) und Diarrhoe (10-30\%). Eine Arbeit berichtet über drei neonatale Fälle in China. Bei diesen zeigte sich Kurzatmigkeit, Husten, Fieber und Gastroösophagealer Reflux [10]. Bei Erwachsenen sind weiters Berichte über neurologische Auffälligkeiten bekannt [20]. Ebenso kann es auch im Rahmen der Infektion zum Auftreten von Hautveränderungen kommen [21].

Bei Erwachsenen beträgt die Zeit bis zur Genesung für leichte Fälle ca. 2 Wochen, während sie für schwerwiegende Fälle mit 3-6 Wochen angegeben wird. Inwieweit sich Inkubationszeit und Erkrankungsdauer bei Kindern von denjenigen bei Erwachsenen unterscheiden, ist noch nicht ausreichend untersucht [22]. In einer Arbeit mit 171 Kindern wird die mediane Fieberdauer mit 3 Tagen und die maximale Dauer mit 16 Tagen angegeben [8]. In einer kleinen Fallkohorte mit sieben Kindern in der frühen Phase der Epidemie in Wuhan zeigten sich Fieberverläufe zwischen 3 und 11 Tagen [7].

Ende April wurde in England eine Warnmeldung aufgrund vermehrter Fälle von Kindern mit einem ungewöhnlichen Inflammationssyndrom veröffentlicht. Die Symptomatik sei ähnlich einem atypischen Kawasaki

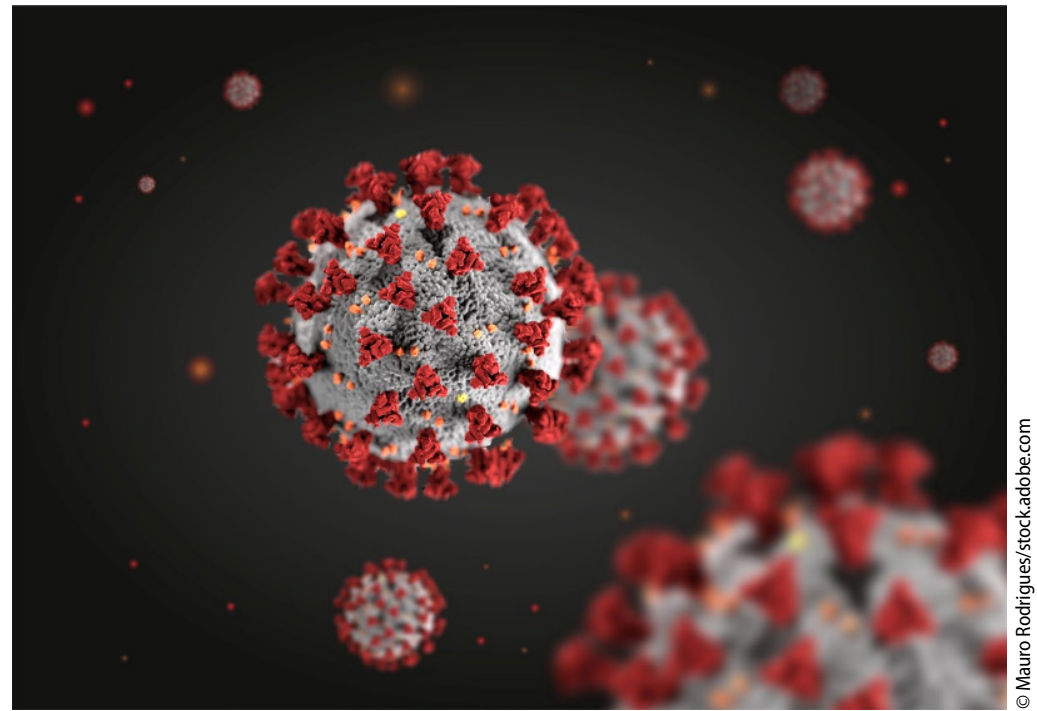

$\Delta$ Coronavirus, strukturelle Morphologie

oder Toxic Shock Syndrom. Im Vordergrund stehen oft Bauchschmerzen und andere gastrointestinale Symptome. Die SARS-CoV-2 PCR war bei diesen Kindern teilweise negativ bei jedoch positiver SARS-CoV-2 Serologie oder Kontakt zu SARS-CoV-2 infizierten Personen [23]. Inzwischen sind solche Fälle auch in anderen Europäischen Ländern (inklusive Österreich) und den USA beobachtet worden. Die Pathophysiologie und die Rolle von SARS-CoV-2 sind bisher unklar.

\section{Labor- und radiologische Befunde}

Laboruntersuchungen bei Kindern zeigten neben normalen oder erniedrigten Gesamt-Leukozyten auch eine Lymphopenie oder Neutropenie. In schweren Fällen kann es zu erhöhten LDH-Werten oder Leberwerten kommen. CRP und PCT sind häufig normal [17], bei Hyperinflammationssyndrom aber stark erhöht [23].

In den durchgeführten $\mathrm{CT}$ Untersuchungen zeigen sich bei Kindern - ähnlich wie bei Erwachsenen -vorzugsweise fleckförmige, milchglasartige Trübungen [18].

\section{Springer Medizin}

\section{Erkrankung bei Kindern} mit Risikofaktoren

Bei Erwachsenen sind als Risikofaktoren für einen schweren Verlauf u.a. kardiovaskuläre Grunderkrankungen, Diabetes mellitus, chronische respiratorische Erkrankungen, arterielle Hypertonie und maligne Erkrankungen (bzw. deren Therapie) genannt $[4,24]$. Daten zu Kindern mit Risikofaktoren und Grunderkrankungen (primäre und sekundäre Immundefizienz, maligne Grunderkrankung, chronische respiratorische Erkrankungen wie Cystische Fibrose, kardiale Erkrankungen, etc.) werden in den bisherigen pädiatrischen Analysen kaum erwähnt [4, 9]. In einer Arbeit mit 171 Kindern mussten drei Kinder intensivmedizinisch behandelt werden, welche unterschiedliche Vorerkrankungen hatten (nicht nähere definierte Hydronephrose, Leukämie in Erhaltungstherapie, Invagination) [8].

Ob sich aus der Tatsache, dass so wenige Erkrankungsfälle bei Kindern mit Grunderkrankungen berichtet werden, ableiten lässt, dass diese weniger gefährdet sind als Erwachsene mit Risikofaktoren, ist in Diskussion. Auch italienische Kollegen berichten lediglich von Einzelfällen von SARS-CoV-2-erkrankten Kindern mit 
oben genannten Risikofaktoren (persönliche Kommunikation). In einem Bericht aus den USA hatten 80 von 354 Kindern zumindest eine Grunderkrankung, wobei die meisten Kinder chronische Lungenerkrankungen wie Asthma ( $n=40)$ oder kardiovaskuläre Erkrankungen $(n=25)$ hatten oder immunsupprimiert waren $(n=10)$ [25].

\section{Testung}

Der Nachweis von SARS-CoV-2 erfolgt mittels RT-PCR. Als Material kann neben einem naso- oder oropharyngealen Abstrich auch Sputum, BAL oder Trachealsekret herangezogen werden. Ein negatives Ergebnis schließt eine Infektion nicht mit Sicherheit aus und sollte bei anhaltendem Verdacht wiederholt werden [26]. Eine Testung vor Symptombeginn kann trotz vorliegender Infektion negativ sein, da es erst ca. 1-2 Tage vor Symptombeginn zur Virus-Ausscheidung kommt [14]. Bei einem Großteil der Patienten ist Virus-RNA mindestens 7 Tage lang im Nasopharynx nachweisbar [27] jedoch gibt es auch Berichte, dass Virus-RNA nur intermittierend im Nasopharynx nachweisbar ist. Es wurde gezeigt, dass die Viruslast zu Symptombeginn im Nasopharynx hoch ist, dann aber rasch abnimmt und eine weitere Virusreplikation im unteren Respirationstrakt stattfindet und dort auch länger nachweisbar ist [28]. Somit gibt es durch den Probenentnahmeort und den Zeitpunkt des Probengewinnes Schwankungen in der Sensitivität. Die Spezifität hingegen ist hoch, da nur SARS-CoV eine Kreuzreaktion zeigt [29]. Bei Kindern wurde auch eine Virusausscheidung im Stuhl beobachtet, wobei bisher keine Hinweise für eine Infektiosität gefunden wurden [18].

Derzeit verfügbare Antikörpertests sind klinisch nur unzureichend validiert, sodass keine robusten Daten zur Sensitivität und Spezifität vorhanden sind [30].

\section{Therapie und Management}

Wichtiger Bestandteil des Managements von bestätigten COVID-19 Patientinnen und Patienten ist die frühzeitige Isolierung zur Verhinderung der weiteren Transmission. Bei stationärer Aufnahme sollte neben strengsten Isolationsmaßnahmen eine regelmäßige Überwachung hinsichtlich des klinischen Zustands und der Vitalparameter (insbesondere der Oxygenierung) erfolgen, um frühzeitig - die bei Erwachsenen oft als plötzlich einsetzend beschriebene - Verschlechterung des Krankheitsverlaufes zu erkennen [31].

Die Datenlage zu spezifischen therapeutischen Maßnahmen insbesondere bei Kindern ist noch sehr schwach. Bisher gibt es keine zugelassenen Medikamente zur Therapie von COVID-19 bei Erwachsenen und Kindern [32]. Eine publizierte Arbeit aus China empfiehlt bei Kindern inhalativ Interferon-alpha-2 sowie Lopinavir/ Ritonavir [31]. In einer randomisiertkontrollierten Studie mit 199 Erwachsenen mit schwerem Verlauf zeigte Lopinavir/Ritonavir jedoch keinen signifikanten Vorteil [33]. Der Einsatz von Kortikosteroiden sollte vermieden werden und nur in speziellen intensivmedizinischen Indikationen in Betracht gezogen werden [31, 34]. Remdesivir, welches ursprünglich zur Therapie von Ebola entwickelt wurde, zeigt in vitro eine Hemmung von SARS-CoV-2 [35] und wurde bei Erwachsenen bereits in einzelnen Fallserien eingesetzt und wird derzeit in klinischen Studien geprüft. Erste Daten aus einer randomisiert-kontrollierten MulticenterStudie zeigten keinen statistisch signifikanten Überlebens-Vorteil durch Remdesivir, auch wenn Patienten unter Remdesivir tendenziell eine kürzere Genesungszeit hatten [36]. Daten zum Einsatz bei Kindern sind jedoch bisher nicht bekannt. (Hydroxy-)Chloroquin zeigt ebenso eine in vitro Aktivität [35, 37], jedoch sind auch hier bisher nur wenige Daten und ausschließlich bei Erwachsenen bekannt. Ein Kind erhielt auf einer Intensivstation intravenöse Immunglobuline [7]. Allerdings ist nicht anzunehmen, dass in handelsüblichen, gepoolten Immunglobulin-Präparaten spezifische Antikörper gegen dieses neuartige Virus enthalten sind, eine immunmodulatorische Wirkung aber nicht ausgeschlossen ist.

Eine Entlassung bzw. Entisolierung sollte erst nach Genesung und dem Nachweis von zwei negativen PCR Tests im Abstand von mindestens 24 Stunden erfolgen [22].

\section{Übertragung auf und von Kindern}

Aufgrund von Daten bei anderen respiratorischen Infektionen (z. B. Influenza) und der milden und eventuell auch asymptomatischen Verläufe bei Kindern, wurde über deren Bedeutung für die Transmission spekuliert. Kinder scheinen aber nicht nur seltener Symptome zu entwickeln, sondern sich möglicherweise auch seltener zu infizieren. Eine Studie zur Transmission innerhalb von Haushalten von Indexpatienten hat gezeigt, dass sich Erwachsene fünfmal häufiger infiziert haben als Kinder [38]. Eine andere, ähnliche Studie konnte diesen Unterschied jedoch nicht zeigen [39]. In einer isländischen Studie zur Rate an asymptomatisch Infizierten waren Erwachsenen doppelt so oft Virusträger (1\%) als Kinder, Jugendliche und Erwachsene zwischen 10 und 20 Jahren $(0,5 \%)$. Von über 800 untersuchten Kindern unter 10 Jahren war kein einziges positiv, obwohl die Grundschulen und Kinderbetreuungseinrichtungen während des Untersuchungszeitraumes geöffnet waren [40]. Ein australischer Bericht zeigt, dass von 18 SARS-CoV-2 Infizierten (9 Schüler und 9 Angestellte) in 15 Schulen nur 2 von ca. 850 engen Kontaktpersonen infiziert wurden [41].

\section{Conclusio}

Bisherige Daten zu pädiatrischen COVID-19 Fällen sind rar, nehmen jedoch von Tag zu Tag zu. Kinder erkranken im Vergleich zu Erwach- 
senen wesentlich seltener und haben meist einen milderen Verlauf. Schwere und kritische Verläufe sind selten, Todesfälle werden vereinzelt berichtet. Ob Säuglinge oder Kinder mit Vorerkrankungen ein höheres Risiko für schwere Verläufe haben, werden weitere Daten zeigen.

Auch wenn Kinder seltener und milder betroffen sind, müssen pädiatrische Einrichtungen auf die Anforderungen dieser Pandemie (Isolationsmaßnahmen, Schutz der Mitarbeiterinnen und Mitarbeiter, Diagnostik, Behandlung, ggf. Intensiv-Behandlung, Management von Patienten mit erkrankten Eltern) vorbereitet sein.

\section{Zurl, W. Zenz, D. S. Kohlfürst,}

\section{Strenger}

Universitätsklinik für Kinder- und Jugendheilkunde, Medizinische Universität Graz, für die AG Infektiologie der ÖGKJ

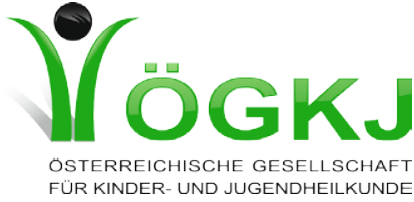

\section{Korrespondenzadresse}

\section{Volker Strenger}

Arbeitsgruppe „Infektiologie“ der

ÖGKJ, Forschungseinheit, Infectious

Diseases in the Immunocompromised

Host", Universitätsklinik für Kinder- und Jugendheilkunde

Medizinische Universität Graz,

Auenbruggerplatz 30 ,

$8036 \mathrm{Graz}$, Österreich

volker.strenger@medunigraz.at

\section{Literatur}

1. Zhu N, Zhang D, Wang W, Li X, Yang B, Song $J$ et al (2020) A Novel Coronavirus from Patients with Pneumonia in China, 2019. N Engl J Med 382(8):727-733

2. Cucinotta D, Vanelli M (2020) WHO Declares COVID-19 a Pandemic. Acta Biomed 91(1):157-160

3. University JH (2020) COVID-19 Dashboard by the Center for Systems Science and Engi-

Hier steht eine Anzeige. 
neering (CSSE) at Johns Hopkins University (JHU). https://coronavirus.jhu.edu/map.html

4. Wu Z, McGoogan JM (2020) Characteristics of and Important Lessons From the Coronavirus Disease 2019 (COVID-19) Outbreak in China: Summary of a Report of 72314 Cases From the Chinese Center for Disease Contro and Prevention. JAMA

5. Ministerio de Sanidad (2020) Actualización n91. Enfermedad por el coronavirus (COVID-19). https://www.mscbs.gob.es/ profesionales/saludPublica/ccayes/alertasActual/nCov-China/documentos/Actualizacion_91_COVID-19.pdf (Erstellt: 30. Apr. 2020)

6. di Istituto Superiore, Sanità R Characteristics of SARS-CoV-2 patients dying in Italy 2020. https://www.epicentro.iss.it/en/coronavirus/bollettino/Report-COVID-2019_29_ april_2020.pdf

7. Liu W, Zhang $\mathrm{Q}$, Chen J, Xiang $\mathrm{R}$, Song $\mathrm{H}$, Shu $S$ et al (2020in) Detection of Covid-19 in Children in Early January 2020 in Wuhan, China. N Engl J Med

8. Lu X, Zhang L, Du H, Zhang J, Li YY, Qu J et al (2020) SARS-CoV-2 Infection in Children. $N$ Engl J Med

9. Dong $Y, M o X, H u Y, Q i X$, Jiang $F$, Jiang $Z$ et al (2020) Epidemiological Characteristics of 2143 Pediatric Patients With 2019 Coronavirus Disease in China. Pediatrics

10. Chen H, Guo J, Wang C, Luo F, Yu X, Zhang $W$ et al (2020) Clinical characteristics and intrauterine vertical transmission potential of COVID-19 infection in nine pregnant women: a retrospective review of medical records. Lancet 395(10226):809-815

11. Zhu H, Wang L, Fang C, Peng S, Zhang L, Chang $\mathrm{G}$ et al (2020) Clinical analysis of 10 neonates born to mothers with 2019-nCoV pneumonia. Transl Pediatr 9(1):51-60

12. Wang S, Guo L, Chen L, Liu W, Cao Y, Zhang $J$ et al (2020) A case report of neonatal COVID-19 infection in China. Clin Infect Dis

13. Zeng H, Xu C, Fan J, Tang Y, Deng Q, Zhang W et al (2020) Antibodies in Infants Born to Mothers With COVID-19 Pneumonia. JAMA

14. European Centre for Disease Prevention and Control (ECDC Novel coronavirus disease 2019 (COVID-19) pandemic: increased transmission in the EU/EEA and the UK - sixth update2020 12 March 2020 18.03.2020. https://www.ecdc.europa.eu/sites/default/ files/documents/RRA-sixth-updateOutbreak-of-novel-coronavirus-disease2019-COVID-19.pdf

15. Cai J, Xu J, Lin D, Yang Z, Xu L, Qu Z et al (2020) A Case Series of children with 2019 novel coronavirus infection: clinical and epidemiological features. Clin Infect Dis

16. Xia W, Shao J, Guo Y, Peng X, Li Z, Hu D (2020) Clinical and CT features in pediatric patients with COVID-19 infection: Different points from adults. Pediatr Pulmonol

17. Zimmermann $P$, Curtis $N$ Coronavirus Infections in Children Including COVID-19: An Overview of the Epidemiology, Clinical Features, Diagnosis, Treatment and Prevention Options in Children. Pediatr Infect Dis J
18. Xu Y, Li X, Zhu B, Liang H, Fang C, Gong Y et al (2020) Characteristics of pediatric SARSCoV-2 infection and potential evidence for persistent fecal viral shedding. Nat Med

19. Wei M, Yuan J, Liu Y, Fu T, Yu X, Zhang Z-J (2020) Novel Coronavirus Infection in Hospitalized Infants Under 1 Year of Age in China. JAMA

20. Helms J, Kremer S, Merdji H, Clere-Jehl $\mathrm{R}$, Schenck M, Kummerlen C et al (2020) Neurologic Features in Severe SARS-CoV-2 Infection. N Engl J Med

21. Recalcati S (2020) Cutaneous manifestation in COVID-19: a first perspective. J Eur Acad Dermatology Venereol

22. World Health Organization (WHO) Report of the WHO-China Joint Mission on Coronavirus Disease 2019 (COVID-19)2020 18.03.2020. https://www.who.int/docs/ default-source/coronaviruse/who-chinajoint-mission-on-covid-19-final-report.pdf

23. Riphagen $S$, Gomez X, Gonzalez-Martinez C, Wilkinson N, Theocharis P (2020) Hyperinflammatory shock in childre during COVID-19 pandemic. Lancet. https:// doi.org/10.1016/S0140-6736(20)31094-1

24. Zhou F, Yu T, Du R, Fan G, Liu Y, Liu Z et al (2020) Clinical course and risk factors for mortality of adult inpatients with COVID-19 in Wuhan, China: a retrospective cohort study. Lancet

25. Coronavirus Disease 2019in Children - United States, February 12-April 2, 2020. MMWR Morbidity and mortality weekly report. 2020;69(14):422-426

26. Robert Koch Institut (RKI) Hinweise zur Testung von Patienten auf Infektion mit dem neuartigen Coronavirus SARS-CoV-2 2020 https://www.rki.de/DE/Content/InfAZ/N/ Neuartiges_Coronavirus/Vorl_Testung_ nCoV.html

27. Young BE, Ong SWX, Kalimuddin S, Low JG, Tan SY, Loh J et al (2020) Epidemiologic Features and Clinical Course of Patients Infected With SARS-CoV-2 in Singapore. JAMA 323(15):1488-1494

28. Wölfel R, Corman VM, Guggemos W, Seilmaier M, Zange S, Müller MA et al (2020) Virological assessment of hospitalized patients with COVID-2019. Nature

29. Corman VM, Landt O, Kaiser M, Molenkamp R, Meijer A, Chu DK et al (2020) Detection of 2019 novel coronavirus (2019-nCoV) by real-time RT-PCR. Euro Surveill 25(3)

30. Österreichische Gesellschaft für Laboratoriumsmedizin und Klinische Chemie Labordiagnostik bei Coronavirus SARS-CoV-22020 30.04.2020. https://www.oeglmkc.at/ down/OeGLMKC\%20Empfehlungen\%20 COVID19_20200417.pdf

31. Chen ZM, Fu JF, Shu Q, Chen YH, Hua CZ, $\mathrm{Li} F B$ et al (2020) Diagnosis and treatment recommendations for pediatric respiratory infection caused by the 2019 novel coronavirus. World J Pediatr

32. Sanders JM, Monogue ML, Jodlowski TZ, Cutrell JB (2020) Pharmacologic Treatments for Coronavirus Disease 2019 (COVID-19): A Review. JAMA
33. Cao B, Wang Y, Wen D, Liu W, Wang J, Fan G et al (2020) A Trial of Lopinavir-Ritonavir in Adults Hospitalized with Severe Covid-19. N Engl J Med

34. CDC Coronavirus Disease 2019 (COVID-19). Information for Pediatric Healthcare Providers 2020 [18.03.2020. https://www.cdc. gov/coronavirus/2019-ncov/hcp/pediatrichcp.html

35. Wang M, Cao R, Zhang L, Yang X, Liu J, Xu $M$ et al (2020) Remdesivir and chloroquine effectively inhibit the recently emerged novel coronavirus (2019-nCoV) in vitro. Cell Res 30(3):269-271

36. Wang Y, Zhang D, Du G, Du R, Zhao J, Jin $Y$ et al Remdesivir in adults with severe COVID-19: a randomised, double-blind, placebo-controlled, multicentre trial. Lancet

37. Yao X, Ye F, Zhang M, Cui C, Huang B, Niu $P$ et al (2020) In Vitro Antiviral Activity and Projection of Optimized Dosing Design of Hydroxychloroquine for the Treatment of Severe Acute Respiratory Syndrome Coronavirus 2 (SARS-CoV-2). Clin Infect Dis

38. Li W, Zhang B, Lu J, Liu S, Chang Z, Cao P et al (2020) The characteristics of household transmission of COVID-19. Clin Infect Dis

39. Bi Q, Wu Y, Mei S, Ye C, Zou X, Zhang Z et al (2020) Epidemiology and transmission of COVID-19 in 391 cases and 1286 of their close contacts in Shenzhen, China: a retrospective cohort study. Lancet Infect Dis

40. Gudbjartsson DF, Helgason $A$, Jonsson $H$, Magnusson OT, Melsted P, Norddahl GL et al (2020) Spread of SARS-CoV-2 in the Icelandic Population. N Engl J Med

41. National Centre for Immunisation Research and Surveillance COVID-19 in schools - the experience in NSW 2020. http://ncirs.org. au/sites/default/files/2020-04/NCIRS\%20 NSW\%20Schools\%20COVID_Summary_FINAL\%20public_26\%20April\%202020. pdf

Hinweis des Verlags. Der Verlag bleibt in Hinblick auf geografische Zuordnungen und Gebietsbezeichnungen in veröffentlichten Karten und Institutsadressen neutral.

Paediatr. Paedolog. 2020 · 55:138-143 https://doi.org/10.1007/s00608 020-00794-1 (c) Springer-Verlag GmbH Austria, ein Tei von Springer Nature 2020 
Hier steht eine Anzeige.

Springer 